\title{
OPTIMIZED MULTI-OBJECTIVE ROUTING FOR WIRELESS COMMUNICATION WITH LOAD BALANCING
}

\author{
Dr. A. Sathesh, \\ Assistant Professor, \\ Department of Electronics and Communication Engineering, \\ Eritrea Institute of Technology, Eritrea. \\ Email: Sathesh4you@gmail.com
}

\begin{abstract}
The rapid advancements in the wireless communication networks have increased the popularity of portable or mobile devices and the network framed with these mobile devices. These mobile networks framed using the volunteering portable devices are decentralized and have dynamic topologies experiencing sudden changes in the network structure. The main reason causing the topology changes are the limited energy availability of the device and their mobility. Improper trafficking of the tasks and improper selection of the portable devices causes maximum energy consumption resulting in the link failures and changes in the topology of the network. So the paper puts forward the hybridized optimization technique to handle the multi-objective problem faced by these decentralized networks. The proposed method is validated using the network simulator- 2 to evince throughput, energy consumption and the network longevity achieved by the proposed method.
\end{abstract}

Keywords: portable devices, decentralized network, proper traffic control, throughput, energy consumption, and network longevity

\section{INTRODUCTION}

Due to the increasing number of mobile terminals it becomes important to develop a more effective routing protocol that retains the topology of the network for a longer time and provide better bandwidth utilization. For the networks framed in the ad hoc manner without any infrastructure the consumption of the energy and the bandwidth exceeds the limit in-order retain the topology of the network. The topology changes occur due to the sudden movement of the device that is used in framing the network or the complete draining of the battery leaving the mobile devices dead.

ISSN: $2582-4104$ 
Journal of trends in Computer Science and Smart technology (TCSST) (2019)

Vol.01/ No. 02 Pages: 106-120

https://www.irojournals.com/tcsst/

DOI: https://doi.org/10.36548/jtcsst.2019.2.004

This causes a vacant space in the network affecting the proper transmission of the data packets, and resulting in the failure of the information transmission or causing partial information transmission or change of destination. The fig. 1 below shows the network framed in the adhoc manner along with the possibility of failures.

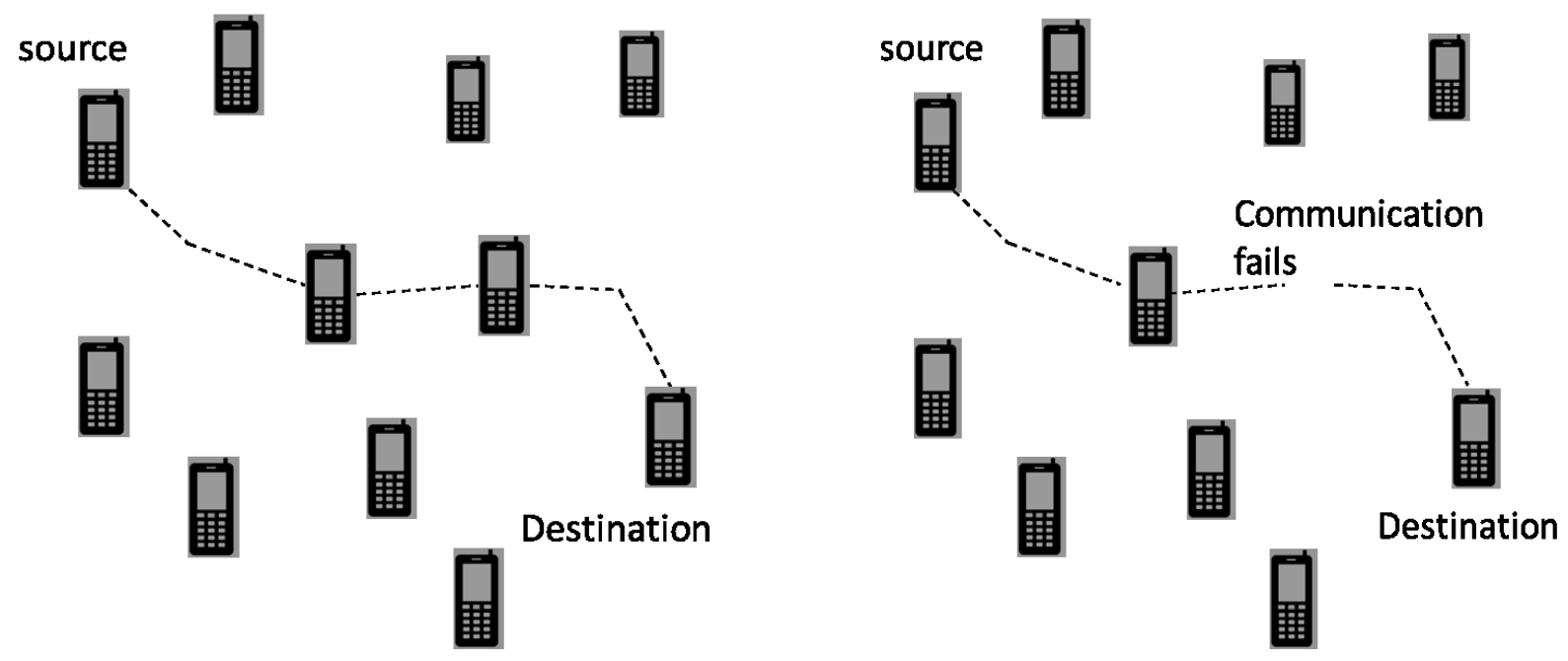

Fig.1 Adhoc Network

The failures affecting the transmission is usually the result of the ineffective routing protocol that fails to identify a path that holds befitting mobile devices that could remain alive and stable throughout the transmission process. During continuous transmission of information even the nodes that are stable with the high battery availability are affected as they are could hold only limited amount of energy. The implementation of the energy harvesting would be beneficial but costly. All these issues result as the multi-objective problem in identifying the path for the decentralized network framed in the adhoc manner.

ISSN: $2582-4104$ 
Journal of trends in Computer Science and Smart technology (TCSST) (2019)

Vol.01/ No. 02 Pages: 106-120

https://www.irojournals.com/tcsst/

DOI: https://doi.org/10.36548/jtcsst.2019.2.004

\subsection{PROBLEM DEFINITION}

The effective routing to be devised needs to identify a node with the optimal energy (opt $t_{\text {energy }}$ ) and the optimal

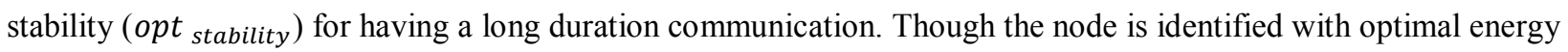
availability, it becomes necessary to identify a path that consumes less energy. As the energy consumption $\left(E_{c}\right)$ is directly proportional to the delay ( delay $_{\text {trans }}$ ) in the transmission of the information and in turn as the delay is related to the distance in the path $\left(\right.$ dist $\left._{p a t h}\right)$ form the source to the destination $\left(d i s t_{S r-D e}\right)$ and distance between the nodes /mobile devices $\left(\right.$ dist $\left._{n-n}\right)$ it becomes necessary for the routing protocol to find out a path with the that has nodes with minimum distance to the destination. The equation (1) below is framed in this regard.

$$
E_{c} \propto \text { delay }_{\text {trans }} \propto \text { dist }_{\text {path }}=\left(\text { dist }_{S r-D e}+\text { dist }_{n-n}\right)
$$

The mobile natures of the devices also remain as an obstacle affecting the transmission in the network, so it is entailed that the routing protocol should identify the more stable nodes for the transmission and this is a tedious task. So it is essential keep note of the nodes leaving and entering the task in each instance and replace the nearest available nodes instead of the dead or the mobile nodes to have a continuous transmission without any interruptions. To have a stable transmission the load balancing $\left(N_{l}\right)$ also becomes essential, so the multi-objective problems to be optimized are the energy and the stability. The equation (2) lists out the multi-objectives (multiobj) to be optimized to make the routing more effective.

$$
\text { multi }_{\text {obj }}=\left\{\begin{array}{l}
O b j_{1}=o p t_{\text {energy }} \\
O b j_{2}=o p t_{\text {stability }}
\end{array}\right.
$$

So the paper utilizes the hybridized optimization algorithm the foraging behavior of the Ant-Lion (AL-O) and the Cuckoo search optimization to identify the most befitting path enriched with the stable nodes with high energy availability.

ISSN: $2582-4104$ 
Journal of trends in Computer Science and Smart technology (TCSST) (2019)

Vol.01/ No. 02 Pages: 106-120

https://www.irojournals.com/tcsst/

DOI: https://doi.org/10.36548/jtcsst.2019.2.004

The remaining paper holds the related works in section 2, the proposed work in section 3, the Result Evaluation in section 4 and conclusion in section 5 followed by the references.

\section{RELATED WORKS}

The related works below details the existing method that were utilized in identifying the optimal route for the communication extension in the wireless medium, most of the researches, identify the energy aware routing and fails to take in to account the mobile nature of the nodes. Some of the researches that have developed the routing protocol with energy optimization included to the related works author Smys, S et al [1] in his paper has put forth an "energyaware routing enumerating the distance between the nodes for the wireless sensor networks and that are utilized in the big data applications such as the social media" and in his previous issue [2] has utilized the "K-connection Maintenance algorithm for Balanced Routing in Mobile Ad Hoc Networks." the author Sathish, et al [3] in his paper puts forth the "clustering techniques utilizing the fuzzy interference and the neural network to frame an energy efficient routing for the IOT-WSN"

The author Duraipandian, et al [4] in his paper proposes a "Routing Algorithm for Manet Based on the Machine Learning Techniques" Raj, Jennifer S et al [5] puts forward a "Energy Efficient Multi-Tier Sustainable Secure Routing Protocol for Mobile Wireless Sensor Networks" utilizing the K-Means clustering along with the ALO based optimization . Natarajan et al [6] analysis the performance of the proactive routing protocol developed for the flying wireless networks.

Vijayalakshmi, et al [7] proposes an effective method of cluster head selection using the Tabu-Particle search algorithm to frame energy optimized routing for the wireless sensors. Dr.AbulBasar et al [8] proposes an energy efficient routing protocol to the for the wireless sensor involved in the internet of things to have an optimized quality of service. Seetha et al [9] presents the "Framework for Multi-Constraint Multicast Routing in Wireless Mesh Networks"

Bhalaji, et al [10] details the "Performance Evaluation of Flying Wireless Network with Vanet Routing Protocol" and the Wilson, et al [11] proposes the "Improved multi objective data transmission using conventional route 109

ISSN: $2582-4104$ 
Journal of trends in Computer Science and Smart technology (TCSST) (2019)

Vol.01/ No. 02 Pages: 106-120

https://www.irojournals.com/tcsst/

DOI: https://doi.org/10.36548/jtcsst.2019.2.004

selection algorithm in mobile ad hoc network." The author John, et al [12] proposes the "Multi-objective Taylor Crow Optimization Algorithm for Cluster Head Selection in Energy Aware Wireless Sensor Network" the author al et al [13] and kumar et al [14] present the survey on the "Routing in Wireless Sensor Networks Using Optimization Techniques" and the "Hybrid Intelligence Based Routing Protocols" and Josemin Bala, et al [15] in her paper presents the protocol to address the mobility management in the wireless network. The above paper finds the nodes based on the residual energy availability, and its distance to the destination. The proposed process in the paper considers both residual energy availability, and its distance to the destination along with the load of the nodes and the mobility of the nodes.

\section{PROPOSED METHODOLOGY}

The optimized multi objective routing for the wireless communication formulated as two phases.

(i). Monitoring Of the Mobile Devices

- Monitoring incoming and Out Going device within the range

- Updating the details to the node information table

(ii). Optimization of the objectives.

- Gather the information's from the nodes

- Determines the befitting neighboring nodes

- Initiates a path discovery

- Determines suitable paths

- $\quad$ updates to the path information table

ISSN: $2582-4104$ 
Journal of trends in Computer Science and Smart technology (TCSST) (2019)

Vol.01/ No. 02 Pages: 106-120

https://www.irojournals.com/tcsst/

DOI: https://doi.org/10.36548/jtcsst.2019.2.004

\subsection{OBJECTIVE FUNCTIONS}

To have a communication that with stands for a longer period, there are certain objectives functions to be determined for all the mobile devices that volunteer in forming the network. They are the energy level $\left(E n_{l e}\right)$ of the mobile devices, the load of the mobile device $\left(l d_{m d}\right)$, and the distance of the device to another device $\left(\right.$ dist $\left._{d-d}\right)$ within the range. Usually the energy consumption for a network formed in the adhoc manner is usually experienced during the process of transmission, receiving, node identification $\left(n_{i d}\right)$ and the route identification $\left(R_{i d}\right)$. The equation (3) shows the energy consumption of the adhoc network for the wireless communication.

$$
E_{c}(t)=E n_{l e}(t-1)-E_{c}{ }^{T}(t)-E_{c}{ }^{R}(t)-E_{c}{ }^{n_{i d}}(t)-E_{c}{ }^{R_{i d}}(t)
$$

Where $E n_{l e}(t-1)$ the energy level remaining in the mobile devices, $E_{c}{ }^{T}(t)$ is the energy required for the transmission and the $E_{c}{ }^{R}(t)$ is the energy required for reception, $E_{c}{ }^{{ }^{i d}}(t)$ and the $E_{c}{ }^{{ }}{ }_{i d}(t)$ are the energy consumption in the node identification and the route identification. The equation (4) shows the load level of the network.

$$
l d_{m d}(t)=l d_{m d}(t-1)+l d_{m d}^{r e}(t-1)+l d_{m d}^{r e}(t)
$$

Where the $l d_{m d}(t-1)$ the initial load of the mobile device, $l d_{m d}^{r e}(t-1)$ the load experienced due to the relaying at time $(t-1)$ and the $l d_{m d}^{r e}(t)$ is the load experienced due to the relaying at time at time $(t)$. Where the $l d_{m d}$ ia defined as shown in the eq (5) below.

$$
l d_{m d}=\frac{A v g_{l d}}{A V g_{\text {space }}+A v g_{\text {ene }}}
$$

Where $A v g_{l d}$ are the average load, and the $A V g_{\text {space }}$ and the $A v g_{\text {ene }}$ are the total space available and the total energy consumed respectively.

ISSN: $2582-4104$ 
Journal of trends in Computer Science and Smart technology (TCSST) (2019)

Vol.01/ No. 02 Pages: 106-120

https://www.irojournals.com/tcsst/

DOI: https://doi.org/10.36548/jtcsst.2019.2.004

So as the load of the device is also indirectly associated with the energy consumption, it becomes essential to optimize the load before the befitting nodes and the route is identified.

\subsection{NODE, PATH DISCOVERY AND INFORMATION CONVEYANCE}

The Methodology initiates with the discovering of the relevant nodes suitable for the information transfer, by monitoring the volunteering devices and the incoming and the outgoing devices and regularly updating them into the node information table. The fig .2 shows the node details monitored.

\begin{tabular}{|c|c|c|c|l|l|}
\hline $\begin{array}{l}\text { NODE ID } \\
\left(\text { Node }_{i d}\right)\end{array}$ & $\begin{array}{c}\text { Location } \\
\left(\text { Node }_{l o c}\right)\end{array}$ & $\begin{array}{c}\text { Energy } \\
\text { Availability } \\
\left(E n_{l e}\right)\end{array}$ & $\begin{array}{c}\text { Load } \\
\left(l d_{m d}\right)\end{array}$ & $\begin{array}{l}\text { Distance } \\
\text { from } \\
\text { neighboring } \\
\text { nodes } \\
\left(\text { dist }_{d-d}\right)\end{array}$ & $\begin{array}{l}\text { Mobility } \\
( \\
\end{array}$
\end{tabular}

Fig.2 Node Details

Now the neighbor list gathered are enumerated for its energy level, load status, mobility and the distance between the nodes to identify the befitting nodes, this is done by engaging the ALO [5] . The ALO using the foraging behavior of the ant-lion estimates the quality of the nodes. The following algorithm in the fig. 3 represents the steps in the enumeration of the befitting nodes.

ISSN: $2582-4104$ 
Journal of trends in Computer Science and Smart technology (TCSST) (2019)

Vol.01/ No. 02 Pages: 106-120

https://www.irojournals.com/tcsst/

DOI: https://doi.org/10.36548/jtcsst.2019.2.004

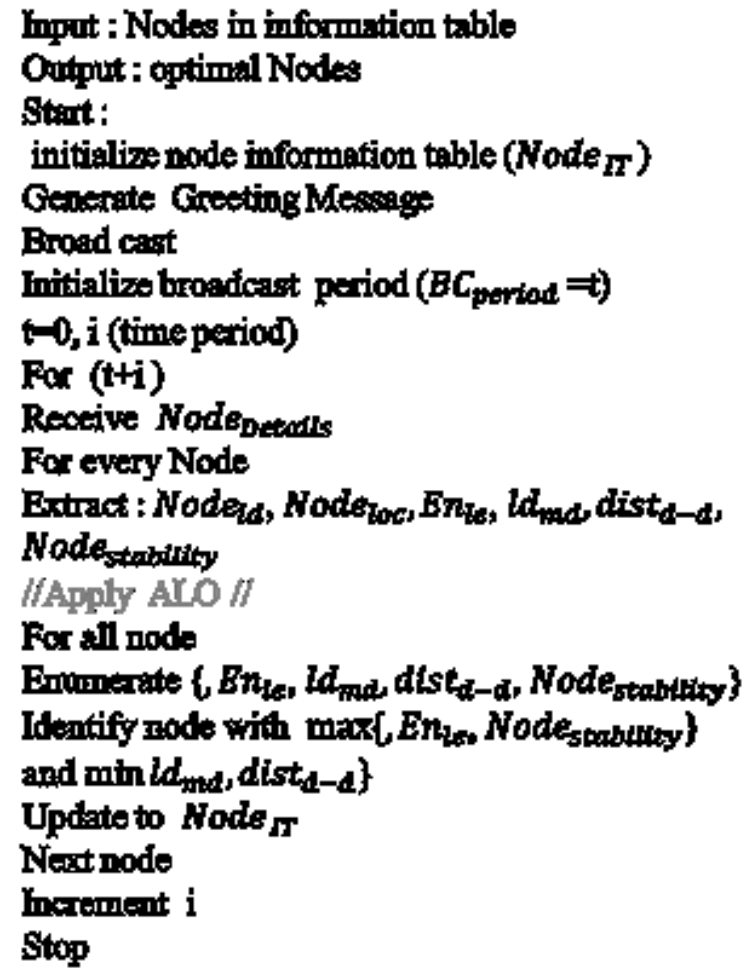

Fig .3 Algorithms for Node Discovery

Once the node more suitable for the conveyance is identified, the path identification is initiated by finding out the most suitable paths that can be used in the transmission using the cuckoo search algorithm [16]. The cuckoo search algorithm. The Algorithm below in fig .4 shows the path discovery process. 
Journal of trends in Computer Science and Smart technology (TCSST) (2019)

Vol.01/ No. 02 Pages: 106-120

https://www.irojournals.com/tcsst/

DOI: https://doi.org/10.36548/jtcsst.2019.2.004

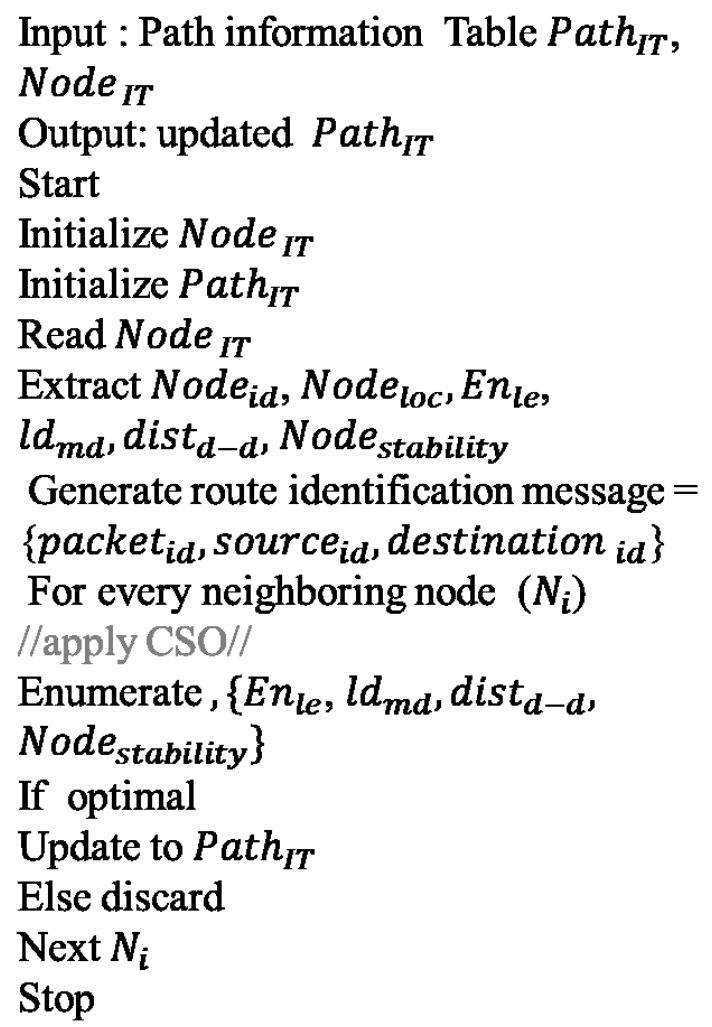

Fig .4 Algorithms for Path Discovery

Based on the information updated in the route identification table the most eligible path is utilized for the information transferring using the greedy face routing methodology $(G F R)$ that utilizes both the proactive and the reactive routing methods. When a need arises to transfer an information from a source $\left(N_{S_{i}}\right)$ to a destination $\left(N_{D_{j}}\right)$, the proactive greedy method of routing is followed to identify a path available from the path information table. If a suitable path is not available, immediately the reactive face routing method is followed to find out a path that is suitable and can with stand the long duration transmission without any failures. Once it identifies the transmission begins. The network is completely monitored at regular intervals to find out the incoming and the outgoing devices. if any of the node engaged in the transmission is found to be dead or leaving the network $\left(N_{l_{n}}\right)$. Immediately the 
Journal of trends in Computer Science and Smart technology (TCSST) (2019)

Vol.01/ No. 02 Pages: 106-120

https://www.irojournals.com/tcsst/

DOI: https://doi.org/10.36548/jtcsst.2019.2.004

alternative node $\left(N_{A_{n}}\right)$ that is close to the $N_{l_{n}}$ is replaced in its position based on the information updated in the node information table.

\section{Result Evaluation}

The Proposed methodology is evaluated using the network simulator-2 for a simulation time of 1000 s with varying number of devices ranging from 100 to 500 involved in the process. The proposed method is enumerated to find out the energy consumption, the throughput and the life time of the network and compared with the EERP [3] and the MOTCO [12] identify the competence of the ALO-CSO in routing the information without any failures. The table.1 below shows the parameters utilized in the simulation.

\begin{tabular}{|l|l|}
\hline \multicolumn{1}{|c|}{ Parameters } & \multicolumn{1}{c|}{ Values } \\
\hline Simulation Time & 1000 seconds \\
\hline Allotted Area & $40 \mathrm{~m} * 2500 \mathrm{~m}$ \\
\hline Number of devices & 500 \\
\hline Packet size & 1024 bits \\
\hline Packet Data Rate & 1 packet per second \\
\hline Initial energy & 100 joules \\
\hline Channel capacity & 2MBPS \\
\hline
\end{tabular}

Table.1 Simulation Parameters

ISSN: 2582-4104 
Journal of trends in Computer Science and Smart technology (TCSST) (2019)

Vol.01/ No. 02 Pages: 106-120

https://www.irojournals.com/tcsst/

DOI: https://doi.org/10.36548/jtcsst.2019.2.004

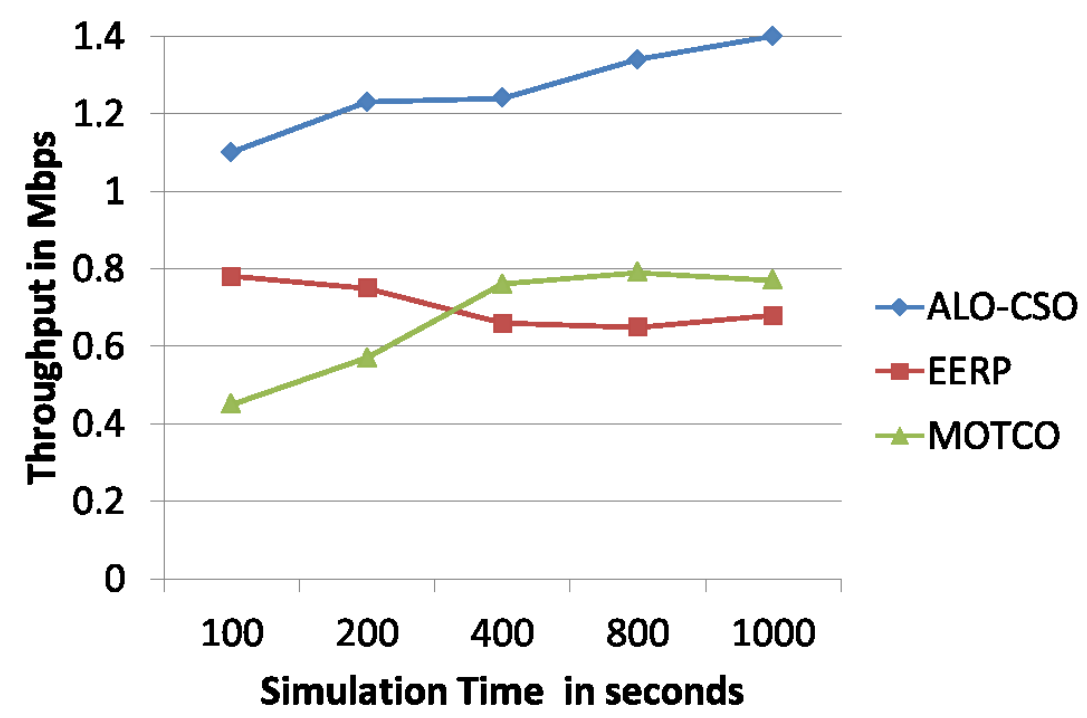

Fig .5 Throughput Measurements

The results above in the fig 5 shows the throughput achieved by the ALO-CSO, the EERP and the MOTCO, the results obtained intimates that the proposed ALO-CSO has 25\% higher throughput than the EERP and $15 \%$ higher throughput than the MOTCO.

ISSN: $2582-4104$ 
Journal of trends in Computer Science and Smart technology (TCSST) (2019)

Vol.01/ No. 02 Pages: 106-120

https://www.irojournals.com/tcsst/

DOI: https://doi.org/10.36548/jtcsst.2019.2.004

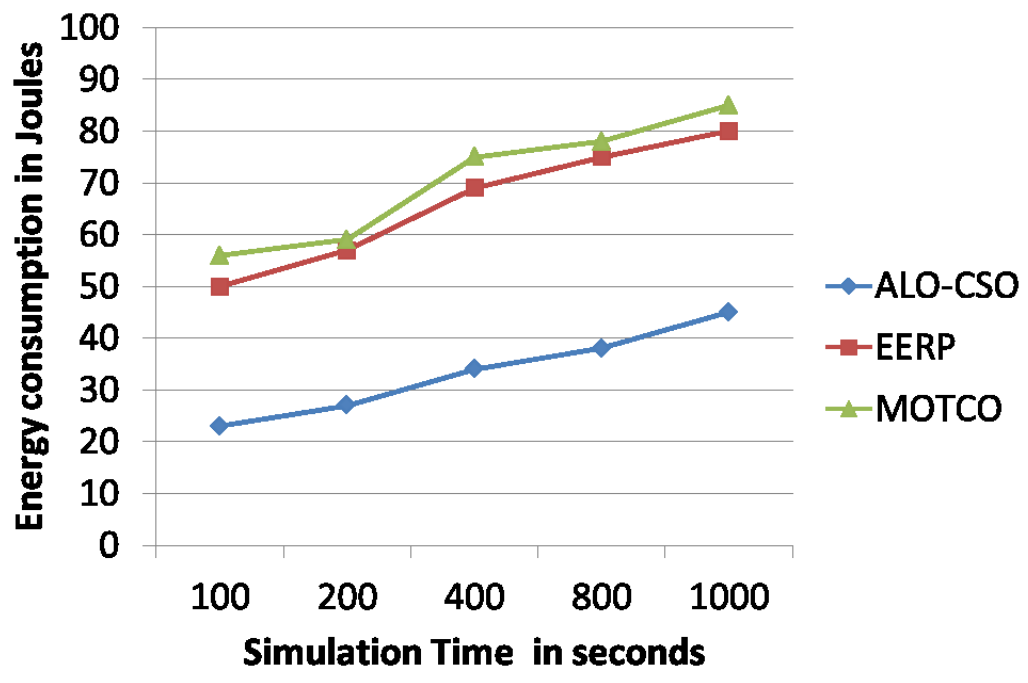

Fig.6 Energy Consumption

The fig. 6 shows the energy consumption of the ALO-CSO, the EERP and the MOTCO and the fig. 8 shows the life time of the network on using the ALO-CSO, the EERP and the MOTCO.

ISSN: $2582-4104$ 
Journal of trends in Computer Science and Smart technology (TCSST) (2019)

Vol.01/ No. 02 Pages: 106-120

https://www.irojournals.com/tcsst/

DOI: https://doi.org/10.36548/jtcsst.2019.2.004

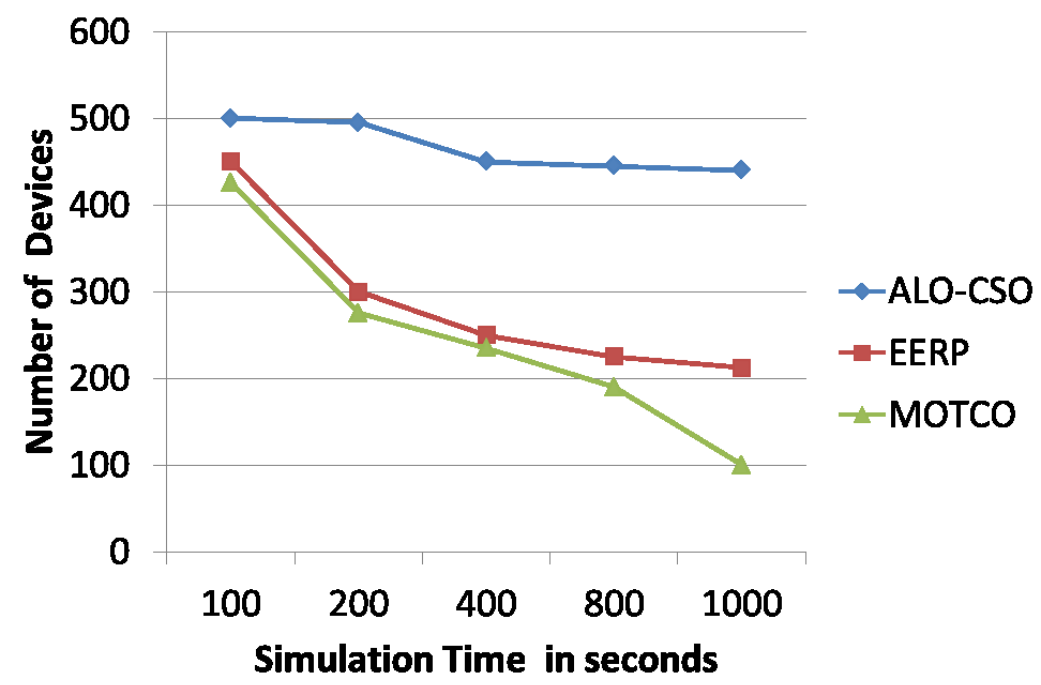

Fig .7 Lifetime of Network

All the above results show that the proposed ALO-CSO has a much high capability than the other two prevailing methods.

\section{CONCLUSION}

The paper in order to have an effective routing with the optimized energy, load and mobility utilizes the ALO-CSO algorithm to find out the suitable devices and the paths for the routing, the method further puts into action the greedy face routing that involves both the proactive and the reactive method of routing to convey the information from the source to the destination. The proposed method is simulated along with the EERP and the MOTCO and the results obtained are compared and the ALO-CSO is found have an improved throughput, energy consumption and network longevity than the prevailing methods. In future the paper is to proceed with secured way of transmission by analyzing the trust of the nodes.

ISSN: $2582-4104$ 
Journal of trends in Computer Science and Smart technology (TCSST) (2019)

Vol.01/ No. 02 Pages: 106-120

https://www.irojournals.com/tcsst/

DOI: https://doi.org/10.36548/jtcsst.2019.2.004

\section{References}

[1] Smys, S. "ENERGY-AWARE SECURITY ROUTING PROTOCOL FOR WSN IN BIG-DATA APPLICATIONS." Journal of ISMAC 1, no. 01 (2019): 38-55.

[2] Smys, S., and G. Josemin Bala. "K-connection Maintenance algorithm for Balanced Routing in Mobile Ad Hoc Networks." International Journal of Computer Networks and Communications (IJCNC) 1, no. 3 (2009): 105-111.

[3] Sathish, "ENERGY EFFICIENT ROUTING IN CLUSTERED IOT WIRELESS SENSORS NETWORKS FOR QOS ENHANCEMENTS" "Journal of ISMAC." no. 01 (2019): 1-11.

[4] Duraipandian, M. "PERFORMANCE EVALUATION OF ROUTING ALGORITHM FOR MANET BASED ON THE MACHINE LEARNING TECHNIQUES." Journal of trends in Computer Science and Smart technology (TCSST) 1, no. 01 (2019): 25-38.

[5] Raj, Jennifer S., S. Smys, and Abul Bashar. "ENERGY EFFICIENT MULTI-TIER SUSTAINABLE SECURE ROUTING PROTOCOL FOR MOBILE WIRELESS SENSOR NETWORKS."

[6] Natarajan, Mr Krishnaraj. "ANALYSIS OF ROUTING PROTOCOLS IN FLYING WIRELESS NETWORKS."

[7] Vijayalakshmi, K., and P. Anandan. "A multi objective Tabu particle swarm optimization for effective cluster head selection in WSN." Cluster computing 22, no. 5 (2019): 12275-12282.

[8] JenniferS.Raj,Dr.AbulBasar"QOS OPTIMIZATION OF ENERGY EFFICIENT ROUTING IN IOT WIRE LESS SENSOR NETWORKS" "Journal of ISMAC." no. 01 (2019): 38-55.

[9] Seetha, S., Sharmila Anand John Francis, E. Grace Mary Kanaga, Esther Daniel, and S. Durga. "A Framework For Multi-Constraint Multicast Routing In Wireless Mesh Networks." In 2019 5th International Conference on Advanced Computing \& Communication Systems (ICACCS), pp. 445-451. IEEE, 2019.

[10] Bhalaji, N. "PERFORMANCE EVALUATION OF FLYING WIRELESS NETWORK WITH VANET ROUTING PROTOCOL." Journal of ISMAC 1, no. 01 (2019): 56-71.

[11] Wilson, J., and Kamalraj Subramaniam. "Improved multi objective data transmission using conventional route selection algorithm in mobile ad hoc network." Peer-to-Peer Networking and Applications (2019): 111.

ISSN: $2582-4104$ 
Journal of trends in Computer Science and Smart technology (TCSST) (2019)

Vol.01/ No. 02 Pages: 106-120

https://www.irojournals.com/tcsst/

DOI: https://doi.org/10.36548/jtcsst.2019.2.004

[12] John, Jacob, and Paul Rodrigues. "MOTCO: Multi-objective Taylor Crow Optimization Algorithm for Cluster Head Selection in Energy Aware Wireless Sensor Network." Mobile Networks and Applications (2019): 1-17.

[13] Al Aghbari, Zaher, Ahmed M. Khedr, Walid Osamy, Ifra Arif, and Dharma P. Agrawal. "Routing in Wireless Sensor Networks Using Optimization Techniques: A Survey." Wireless Personal Communications (2019): 1-28.

[14] Kumar, Dilip, and Tarunpreet Kaur. "Hybrid Intelligence Based Routing Protocols in Wireless Sensor Networks: A Survey." International Journal of Sensors Wireless Communications and Control 9, no. 1 (2019): 2-15.

[15] Smys, S., G. Josemin Bala, and S. Jennifer. "Mobility management in wireless networks using power aware routing." In 2010 International Conference on Intelligent and Advanced Systems, pp. 1-5. IEEE, 2010.

[16] Gandomi, Amir Hossein, Xin-She Yang, and Amir Hossein Alavi. "Cuckoo search algorithm: a metaheuristic approach to solve structural optimization problems." Engineering with computers 29, no. 1 (2013): 17-35.

ISSN: 2582-4104 